\title{
Experience with an extended-release opioid formulation designed to reduce abuse liability in a community-based pain management clinic
}

This article was published in the following Dove Press journal:

International Journal of General Medicine

16 September 2011

Number of times this article has been viewed

\section{Daniel Rubino}

Pain Center of Devon,

Devon, PA, USA
Context: With the growing public health concern over rising rates of opioid abuse, physicians have a responsibility to incorporate safeguards into their practice to minimize the potential for opioid misuse, abuse, and diversion. Patient-specific treatment regimens should include steps to monitor treatment success with regard to optimal pain management as well as inappropriate use of opioids and other substances. Opioid formulations designed to be less attractive for abuse are also being developed. While future studies are needed to determine the impact of such formulations in addressing the issue of opioid misuse in the community as a whole, the experience of practitioners who have utilized these formulations can highlight the practical steps to incorporate such formulations into the everyday patient-care setting.

Purpose: The purpose of this report is to describe experience in managing patients with chronic, moderate-to-severe pain using morphine sulfate and naltrexone hydrochloride extended release capsules (MS-sNT) $\left(\right.$ EMBEDA $^{\circledR}$, King Pharmaceuticals ${ }^{\circledR}$ Inc, Bristol, TN, which was acquired by Pfizer Inc, New York, NY, in March 2011), a formulation designed with features to deter abuse/misuse, in a community-based pain management clinic.

Case presentations: Case reports demonstrating a clinical management plan for assessment, initial interview procedures, explanation/discussion of proposed therapies, patients' treatment goals, conversion to MS-sNT, and titration and treatment outcomes are provided.

Results: The management approach yielded successful outcomes including pain relief, improved quality of life, treatment satisfaction, and patient acceptance of a formulation designed to deter abuse/misuse

Discussion: The cases presented demonstrate that the communication accompanying complete pretreatment assessment, goal-setting and expectations, and attention to individual patient needs can enable optimization of pain-related outcomes, resulting in improved quality of life for patients and fostering patient acceptance of formulations designed to help address opioid abuse/misuse issues in the community at large.

Keywords: morphine abuse, universal precautions, drug abuse, pain management

\section{Introduction}

Opioids play an important role in the management of chronic, moderate-to-severe pain in carefully selected and monitored patients. ${ }^{1,2}$ Opioid therapy may be considered for some patients who cannot achieve pain relief or obtain as favorable a benefit-to-harm evaluation with alternative therapies, and may be part of a multimodal treatment plan. ${ }^{1}$ As chronic pain negatively affects physical functioning, sleep pattern, social activity, and occupational productivity, contemporary pain management focuses on reducing pain while improving functional ability and psychological wellbeing. ${ }^{1-3}$ Knowledge of how pain is affecting the patient can help physicians develop and/or 
refine a patient-specific treatment strategy, determine whether referral to a pain specialist or other specialist is necessary, and set realistic treatment goals. ${ }^{3}$

In addition to the challenges of developing a patientspecific opioid treatment regimen, clinicians must responsibly prescribe in the context of the current growing public health concern over soaring rates of opioid abuse. ${ }^{4}$ In the United States during 2009, among those aged 12 years or older, 2.2 million persons initiated illicit drug use with pain relievers, a rate surpassed only by those initiating with marijuana (2.4 million). ${ }^{5}$ Studies performed among patients being treated for chronic pain reveal rates of opioid and illicit drug abuse that vary widely $(\sim 3 \%-32 \%) .{ }^{6-9}$ While the Federation of State Medical Boards of the United States encourages the use of opioids when appropriate for patients with pain, it also notes the responsibility of physicians to incorporate safeguards into their practices to minimize the potential for their abuse and diversion. ${ }^{10}$

Therefore, a major challenge for the practitioner is to optimize pain management, using opioids when appropriate, by taking steps to both monitor treatment success and assess for inappropriate use of opioids or other substances.

Current guidelines for pain management include several recommendations for clinicians based on the universal precautions approach, which recognizes that it is not always possible to determine which patients misuse, abuse, and divert prescription opioids or those who are likely to do so. ${ }^{11,12}$ Such an approach universally used with all patients may decrease the number of opioid abuse problems that go unrecognized and can reduce the stigma that patients may feel about the types of questions asked at an initial physicianpatient interview ${ }^{11}$ or a request, for example, to undergo a urine drug screen (UDS). An important component of a pain management plan, both to optimize pain management and minimize risks for misuse, abuse, and diversion of opioid analgesics, is patient education and communication. ${ }^{1}$

Formulations that are less attractive for misuse, abuse, and diversion are also being developed by several pharmaceutical companies and may be used as part of a comprehensive risk management plan by providing one step in discouraging prescription opioid misuse, abuse, and diversion. ${ }^{13,14}$ The morphine sulfate and naltrexone hydrochloride extended release capsule (MS-sNT) $\left(\right.$ EMBEDA $^{\circledR}$, King Pharmaceuticals ${ }^{\circledR}$ Inc, Bristol, TN, which was acquired by Pfizer Inc, New York, NY, in March 2011) is indicated for the management of moderateto-severe pain when a continuous, around-the-clock opioid analgesic is needed for an extended period of time. ${ }^{15} \mathrm{MS}-\mathrm{sNT}$ consists of pellets of extended-release morphine sulfate, each containing a core of sequestered naltrexone, an opioid antagonist. ${ }^{15,16}$ Taken as directed, the morphine provides analgesic relief, while the naltrexone remains sequestered, ${ }^{17}$ but, if tampered with by crushing, the naltrexone is released and available to mitigate the morphine-induced subjective effects. ${ }^{16,18}$ Studies performed in nondependent recreational opioid users have indicated that the quantity of naltrexone released upon tampering is sufficient to mitigate the desired subjective effects of the morphine when administered orally or intravenously. ${ }^{16,19}$ While similar studies have not been completed in opioid-dependent recreational users, product labeling and case reports published on two opioid-dependent patients indicate that consumption of MS-sNT that has been tampered with by crushing or chewing may result in opioid withdrawal. This is an important caution for patients prescribed MS-sNT. It is not known whether knowledge of the potential for withdrawal symptoms might further deter individuals who might misuse or abuse MS-sNT. ${ }^{20,21}$

The objective of this report is to describe a successful approach to managing referred patients with chronic, moderate-to-severe pain with inadequate response to previous opioid therapy, focusing on education and communication while using MS-sNT.

\section{Case reports Case I}

A 44-year-old woman with active breast cancer undergoing radiation and chemotherapy had uncontrolled generalized bone pain, particularly sternum. The patient had been on escalating doses of oxycodone $\mathrm{HCl}$ controlled-release tablets ${ }^{22}$ for more than 2 years from previous treating physicians, and at the time of her visit to the pain center had been prescribed controlled-release oxycodone $60 \mathrm{mg}$ every 8 hours and immediate-release oxycodone $5 \mathrm{mg}$ on an as-needed basis as rescue medication for breakthrough pain. The patient had been using 15 tablets of rescue medication per day, exceeding the usual adult dosage of $5 \mathrm{mg}$ every 6 hours. ${ }^{23}$ There had been no subjective evidence of improvement in pain scores or increases in functional mobility or quality of life. Review of medical records indicated that her pain scores, evaluated using a visual analog scale (VAS) $(0-10 ; 0=$ no pain, $10=$ pain as bad as you can imagine), fluctuated, ranging from $4 / 10$ to $10 / 10$; the patient occasionally visited the emergency department for severe pain. The patient was increasingly frustrated and scared, and occasionally confused the dosing schedule by mixing up the terms for immediate-release and controlled-release oxycodone and took the wrong one. The patient also had been previously prescribed citalopram 
$10 \mathrm{mg}$ qd for depression and remained on this medication. She had not had any other treatments or modalities before coming to the pain center. There was no reported history of opioid misuse or abuse.

On her first visit to the pain center, the patient was accompanied by her daughter and a mutual friend. The patient indicated that her goals were to enhance her quality of life, be more active with her children, and improve her overall functional mobility. At this visit, a significant amount of time was spent educating the patient on opioids in general and specifically on the proposed new therapy with MS-sNT, as to the rationale of the formulation, proper use, and compliance. This counseling and education about her medications helped alleviate the patient's considerable apprehension and anxiety over fear of more pain related to the proposed opioid rotation. A point-of-service, qualitative drug screen was performed, which was positive for prescribed opioids and negative for other substances. A written treatment agreement outlining expectations, responsibilities, and boundaries (discussed below) was signed. Oxycodone extended-release therapy was discontinued that day. The patient was instructed to begin taking MS-sNT $100 \mathrm{mg}$ every 12 hours beginning the next morning. She also was instructed to immediately begin taking newly prescribed immediate-release oxycodone $15 \mathrm{mg}$ as rescue medication for breakthrough pain when needed. She was asked to try to limit rescue medication dosing to a maximum of three tablets per day.

At her return visit 6 days later, the woman reported less pain and a VAS score of $2 / 10$, with occasional breakthrough episodes with a VAS score approaching $8 / 10$. The patient reported improved ambulation with less limp and no significant side effects. Her energy level had improved, and she was more active at home. Her daughter was present at this visit and concurred. Planned follow-up included recording of pain assessments using the Pain Assessment and Documentation Tool (PADT), ${ }^{24}$ monthly evaluations for possible side effects, improvements in activities of daily living and functional mobility, referrals to other specialty physicians, if applicable, and adjustment of MS-sNT dose or adjunct medication.

\section{Case 2}

A 50-year-old woman with severe lumbar degenerative disc disease and degenerative joint disease, postlaminectomy syndrome and bipolar disorder had been treated previously with fentanyl transdermal patch $150 \mu \mathrm{g}$ every 48 hours and acetaminophen-hydrocodone 6-8 times per day as rescue medication. VAS pain scores were from $7 / 10$ to $8 / 10$. Medical records were negative for indications of opioid misuse or abuse. During her interview, the patient expressed that her goal was to be more functional in society, be able to go food shopping without being in agony, and be more active with her family. She was provided with information about opioids in general and specifically on the purpose and proper use of MS-sNT. Point-of-service qualitative UDS was performed, with results consistent with treatment history, and the written treatment agreement was signed. Acetaminophen-hydrocodone was discontinued that day; MS-sNT was started the next morning at $80 \mathrm{mg}$ every 12 hours. Also, morphine sulfate immediate release $15 \mathrm{mg}$, up to a maximum of four tablets per day, was to be taken as needed for breakthrough pain.

The patient returned 5 days later accompanied by her sister. She reported no side effects but thought that her pain relief was inadequate. Her dosage of MS-sNT was increased to $100 \mathrm{mg}$ every 12 hours. At a follow-up visit 10 days later, the woman reported good pain control, a VAS score of 4/10, and no side effects. She said that she only had to use two or three doses daily of the rescue medication. She had resumed aquatic therapy, had begun being more active, and indicated that her quality of life had improved significantly. At this follow-up visit, she was accompanied by a family member who agreed and was very pleased with her improvement. The follow-up plan, as in the preceding case, included monthly evaluations and modifications to the treatment plan as warranted.

\section{Case 3}

A 44-year-old man who is a restaurant chef with progressing renal cancer in chemotherapy, complicated by degenerative joint disease of the lumbar spine, was referred to the clinic for pain management. The patient complained of chronic pain in all joints, sternum, burning about the skull, and occasional nausea and fatigue from the chemotherapy. At the time of his referral, his medications included controlledrelease oxycodone $40 \mathrm{mg}$ bid, oxycodone/acetaminophen ${ }^{25}$ $325 \mathrm{mg} / 10 \mathrm{mg}$ 6-8 times daily, and carisoprodol $250 \mathrm{mg}$ bid. ${ }^{26}$ The patient's VAS pain score was still 7/10, confirming that his pain was not relieved. The patient's treatment goal was to be more productive and have better concentration at work, as well as to improve his quality of life.

The patient was rotated to MS-sNT $20 \mathrm{mg}$ bid, oxycodone/acetaminophen was discontinued, and oxycodone $15 \mathrm{mg}$ was prescribed for breakthrough pain. After 1 week, the patient reported no ill effects or adverse events (AEs), but still complained of pain at a VAS score of 7/10. The dose of MS-sNT was increased to $30 \mathrm{mg}$. One week later the patient 
returned to the clinic stating that he was taking 6-8 doses of oxycodone for breakthrough pain and had cut back on his work hours due to pain. The patient was counseled to try to keep the dosing of breakthrough medications to a minimum and the MS-sNT dose was increased to $50 \mathrm{mg}$ bid.

One month later, significant improvement was noted; the patient had reduced his breakthrough medication to three per day, he was again working full time, his VAS score had decreased to $5 / 10$, and there was notable improvement in his affect and mood. No AEs were reported. In subsequent visits, the patient complained of difficulty swallowing, which he attributed to his chemotherapy and disease state. He was shown how to break open a capsule of MS-sNT, and sprinkle the contents over applesauce. After being reminded of the consequences of chewing MS-sNT, the patient was instructed to swallow the applesauce and sprinkles without chewing. After a few days of following this ritual, the patient resumed swallowing the intact MS-sNT in the usual manner. The patient has remained on this therapy with acceptable results while continuing to receive chemotherapy and engage in full-time work.

\section{Discussion}

In the three cases presented, patients had chronic pain that had been difficult to manage prior to referral. The patients presented with pain scores ranging from 4 to 10 , despite opioid therapy and use of rescue medications as many as 6-8 or 15 times per day. Pain was interfering with their activities of daily living and negatively affecting their quality of life. A key component to management of chronic pain is communication, beginning with the history and first consultation visit, to ensure that both patient and provider are comfortable with the treatment plan and approach. ${ }^{12}$ At the Pain Center of Devon, treatment entails a restructuring of the treatment regimen, identification of and agreement on treatment goals, monitoring for aberrant drug-related behaviors, and education about the properties of MS-sNT. The following are typical steps for the management of chronic, moderate-to-severe pain in patients who come to the clinic (Table 1).

\section{Prior to the initial visit}

Management begins prior to the patient visit, when records from the referring and other health care providers are reviewed. These records include progress notes, diagnostic workup, compliance history, illicit drug use, and the clinically judged necessity for opioid analgesics, which are vital to setting a patient's treatment baseline, documenting the rationale for using an opioid, and formulating the treatment plan. ${ }^{10}$

\section{Initial visit}

\section{Review records}

When the patient comes for the initial visit, records are reviewed again, face-to-face, between the physician and patient. ${ }^{1,10,11}$ Many of these patients have been on high doses of medications (most commonly controlled-release oxycodone) for years, but they still have a high degree of pain and other complaints. In some cases, they may need adjunctive therapy, as in the case of neuropathies.

\section{Set baseline and formulate treatment plan, establish treatment goals}

At this initial visit, the patient is given a physical examination and patient-appropriate workup to determine concurrence with the diagnostic reports, set patient baseline, and formulate a treatment plan. In patients who have previously received opioid therapy, this information is useful for ascertaining risk for aberrant, opioid-related behaviors. If a patient is opioid naïve, screening tools can be used to assist in identifying the existence of or risks for opioid misuse, abuse, and addiction, as well as the appropriateness of opioid therapy initiation. ${ }^{13}$

The workup also includes the assessment and management of opioid-associated complications, such as the measurement of hormonal levels. ${ }^{27,28}$ For example, at the Pain Center of Devon, testosterone levels (free, total, and dihydroepianderosterone), which are measured in all male patients who have been on long-term opioid therapy, are usually low. Hormone replacement therapy can be considered and initiated for these patients.

The physician's discussion with the patient includes treatment goals to determine what is most important to the patient, what is most attainable, what physical level the patient hopes to attain within the next year, and what issues the patient is having with pain. The overall goal is to regain function at an opioid dose that is without intolerable side effects. ${ }^{1,10}$ Woven into the discussion is the universal precautions approach to pain management, with the understanding that it is not always possible to predict which patients may develop problematic opioid use. The possibility of diversion by others is also discussed, and patients are encouraged to keep medications in a locked cabinet or safe. Such an approach is intended to maximize patient care and minimize risk of misuse, abuse, and diversion of opioids. ${ }^{11}$

Patients are requested to undergo urine drug screening. A point-of-service qualitative UDS is performed for all patients, and samples are sent out for quantitative analysis for metabolite confirmation. Urine drug screening can aid in determining patient compliance with prescribed opioid therapy and 
Table I Summary of steps used at the Pain Center of Devon for management of chronic moderate-to-severe pain and documentation of clinical decisions

\begin{tabular}{|c|c|c|}
\hline Visit & Steps & Purpose \\
\hline $\begin{array}{l}\text { Prior to initial } \\
\text { visit }\end{array}$ & Review past medical records. & $\begin{array}{l}\text { Set baseline; determine total current daily opioid and rescue medication } \\
\text { use; document rationale for opioid use; formulate treatment plan. }\end{array}$ \\
\hline \multirow[t]{8}{*}{ Initial visit } & Review records again with patient. & Establish physician-patient relationship/partnership in pain management. \\
\hline & $\begin{array}{l}\text { Conduct thorough patient assessment, } \\
\text { including physical examination. }\end{array}$ & $\begin{array}{l}\text { Document/verify medical history; determine current pain intensity } \\
\text { and locations; assess and manage opioid-associated complications. }\end{array}$ \\
\hline & Determine patient's treatment goals. & $\begin{array}{l}\text { Provide opportunity to work with patient to develop } \\
\text { reasonable, achievable goals. }\end{array}$ \\
\hline & Administer UDS with confirmatory test. & $\begin{array}{l}\text { Identify aberrant drug-related behaviors; appropriateness } \\
\text { of initiating opioid therapy. }\end{array}$ \\
\hline & Discuss risks/benefits of opioid therapy. & Provides patient education and awareness. \\
\hline & $\begin{array}{l}\text { Describe proposed new medication and } \\
\text { provide specific instructions for use, purchase } \\
\text { from single physician/pharmacist, and storage. }\end{array}$ & $\begin{array}{l}\text { Provide patient education and awareness of expectations for opioid } \\
\text { use in general and specific features of proposed new medication, } \\
\text { including potential effects of ingesting tampered product. }\end{array}$ \\
\hline & Have patient sign a treatment agreement. & $\begin{array}{l}\text { Establish expectations, responsibilities, boundaries; reinforce physician } \\
\text { directives for opioid use; may help increase patient compliance. }\end{array}$ \\
\hline & $\begin{array}{l}\text { Encourage inclusion of friend/family member } \\
\text { in discussions. }\end{array}$ & $\begin{array}{l}\text { Provides confirmation and elaboration of patient report; } \\
\text { support for patient. }\end{array}$ \\
\hline \multirow[t]{3}{*}{ Conversion } & $\begin{array}{l}\text { Switch overnight to half of equianalgesic dose } \\
\text { of MS-sNT; immediately provide newly prescribed } \\
\text { immediate-release opioid for breakthrough pain. }\end{array}$ & $\begin{array}{l}\text { Immediately addresses patient's need for management } \\
\text { of inadequate pain. }\end{array}$ \\
\hline & $\begin{array}{l}\text { Reevaluate and adjust dose } \\
\text { every } 5-7 \text { days, if needed. }\end{array}$ & $\begin{array}{l}\text { Adjust dose for inadequate pain; address occurrence } \\
\text { of side effects. }\end{array}$ \\
\hline & & Assesses efficacy and AEs. \\
\hline \multirow[t]{3}{*}{ Follow-up } & $\begin{array}{l}\text { Monthly longitudinal monitoring } \\
\text { for compliance. }\end{array}$ & $\begin{array}{l}\text { Documents compliance, effectiveness, side effects, functional } \\
\text { improvements, progress toward goals, and success of treatment. }\end{array}$ \\
\hline & $\begin{array}{l}\text { Have patient fill out PADT form. } \\
\text { Physician completes section on potential } \\
\text { aberrant drug-related behavior. }\end{array}$ & $\begin{array}{l}\text { Provides convenient ongoing assessment and quick review of pain } \\
\text { management over time, including, pain relief, functional changes, mood, sleep, } \\
\text { AEs, progress towards goals, and documentation of drug-related behaviors. }\end{array}$ \\
\hline & $\begin{array}{l}\text { Examine confirmatory results from UDS; } \\
\text { counsel if results are unexpected. } \\
\text { Reevaluate and consider nonopioid } \\
\text { therapies when appropriate. }\end{array}$ & $\begin{array}{l}\text { Determines whether to continue treatment or } \\
\text { whether discontinuation/referral is needed. }\end{array}$ \\
\hline
\end{tabular}

Abbreviations: AE, adverse event; MS-sNT, morphine sulfate and naltrexone hydrochloride extended release capsule; PADT, Pain Assessment and Documentation Tool; UDS, urine drug screen.

detecting use of illicit substances and the presence of opioid before opioid therapy is initiated. ${ }^{1,11,12}$

To allay any fear of potential addiction, it is important for patients receiving opioid therapy for the management of chronic pain to understand the differences between opioid addiction and tolerance, and what potential effects they might experience during opioid treatment. Tolerance is a normal physiological state of adaptation in which exposure to a given dose of drug induces reduced effects over time. ${ }^{29,30}$ This may necessitate dose increases to maintain effectiveness, ${ }^{15}$ while addiction is characterized by behaviors including compulsive use, impaired control over drug use, continued use despite harm, and craving. ${ }^{29,30}$

The patient is then made aware of possible risks (such as opioid-associated AEs, hyperalgesia, and hormonal changes) and benefits associated with opioid therapy. ${ }^{1,11,12}$ The patient is educated on the general management of pain using extendedrelease opioids, with an emphasis on the need to strictly adhere to the dosing schedule so that potentially serious consequences associated with overdosage can be avoided. ${ }^{15}$

\section{Educate patient about proposed therapy}

The features of the MS-sNT formulation, and risks and benefits associated with its use are reviewed. The purpose of the sequestered naltrexone within the MS-sNT formulation is explained to the patient. If the product is tampered with (crushed, chewed, or dissolved), both the morphine and naltrexone are rapidly available. ${ }^{15}$ The dangers of consuming tampered MS-sNT, which may precipitate opioid withdrawal of extremely variable nature and severity in opioid-tolerant 
patients, are emphasized..$^{20,21}$ Patient education is most important to assure patient awareness of potential effects of ingestion of tampered product.

\section{Encourage patient to bring a companion}

Patients are encouraged to bring a friend or family member to all clinic visits to serve as support for the patient and an observer for any concerns. Since patient self-reports can be unreliable, involvement of a family member and/ or caregiver as a reliable observer in assessment of the patient's progress during opioid therapy may help verify and document assessments of functionality, treatment outcomes, and illicit drug use, and may help monitor and respond to the occurrence of AEs. ${ }^{1,11}$ The dialogue that usually ensues with a patient's friends or family members on a follow-up visit includes what the observer witnesses in their interactions with the patient. Such observations might include any observable changes in mentation, behavior, drowsiness, instability, or in the case of conversion to another opioid, any improvements in these side effects that may have been present with the previous medication. Observations from a patient's friend or family member may include any improvements noticed in daily functioning, and quality of life in general. A mate can report any improvements in sleep patterns, frequency of getting out of the house, or complaints about pain.

\section{Treatment agreement}

After receiving instructions and discussing the treatment plan and goals, the patient is asked to sign a treatment agreement (Appendix Figure 1) that documents expectations, responsibilities, and boundaries that patient and physician have agreed upon, including the use of one physician and one pharmacy for medication, and the possibility of discharge from the practice due to certain infractions in the agreement. ${ }^{10}$ The written treatment agreement used in this practice is that from a consensus of several medical organizations dedicated to pain management. ${ }^{31}$ Patients who do not comply with the universal precautions approach and signed treatment agreement are not accepted or treated at the clinic.

Protection of the community is the rationale for using a treatment agreement in patients considered for opioid therapy. The Federation of State Medical Boards of the United States expects physicians to incorporate safeguards into their practices to minimize the potential for abuse and diversion of opioids. ${ }^{10}$ Among the consequences of prescription opioid abuse are increased cost to patients and health care organizations and increased use of health care and criminal justice resources. ${ }^{32,33}$ When offered the explanation that such safeguards may keep prescriptions out of the hands of abusers, the author's experience has been that patients have been supportive of this goal and understanding of the process. The clinic has not experienced difficulty with getting MS-sNT approved with managed care providers.

\section{Conversion}

The patients described in the case reports presented here were converted to MS-sNT because they had inadequate pain control with their previous opioid therapy. The strategy of opioid conversion is intended to achieve a better balance of benefits to harms by switching from one opioid to another. ${ }^{1,34,35}$ The rationale and plan to switch opioids as well as what might be expected during this process is explained to the patients, who often express concern about the occurrence of withdrawal upon discontinuing their medication. Ideally, the starting dose of the new opioid should be sufficient to prevent withdrawal and produce no worsening of pain, but low enough to avoid side effects. ${ }^{34}$

At the Pain Center of Devon, patients are switched overnight to half of an equianalgesic dose of MS-sNT and immediately begin taking newly prescribed, immediaterelease opioid for breakthrough pain. Doses of MS-sNT are started low because, based on experience, it is preferable to make adjustments due to inadequate pain relief than be required to titrate downward. The occurrence of disturbing side effects can increase patient anxiety and cause the patient to be reluctant to use the new medication.

During titration to an effective dose, patients should expect to use about 3 doses of breakthrough medication per day, although more may be needed occasionally. The clinic prefers a titration frequency of every 5-7 days; the patient is provided with an MS-sNT prescription for 5-6 days of medication only and is scheduled for reevaluation in 5-6 days (with early phone-in reports of efficacy, adverse effects, and progress). For safety, a patient starting a new opioid medication or an increasing dose is warned about potential cognitive impairment that may affect driving or work, and the patient is reminded that MS-sNT or any opioid should not be consumed with alcohol. ${ }^{1,15}$

\section{Follow-up visits}

Management procedures for patients with chronic pain who are converted to MS-sNT are the same as with any opioid. Subsequent monthly longitudinal monitoring for compliance, functional mobility improvements, and side effects, among other parameters, is essential for proper management. ${ }^{1}$ Pain 
relief and patient goals (improvements in activities of daily living and functional mobility) are reviewed to determine what progress has been made and whether the treatment plan is still appropriate or needs adjustment. During some visits, the PADT questionnaire, a clinician-directed interview, is administered to the patient. The PADT encompasses assessments of analgesia, activities of daily living, AEs, and potential aberrant drug-related behavior. The section of the PADT concerning potential aberrant drug-related behavior is completed by the physician. ${ }^{24}$ In the clinic, the PADT is administered every 6-12 months. At other visits, the patient is asked about improvements and changes in functional capacity, sleep, mood, and constipation since the previous visit. Patients are again encouraged to include family and/or friends to confirm the current situation and offer input. ${ }^{10}$

If pain relief is inadequate, appropriate adjustments of medications including adjunct medications are made. If pain fails to respond to opioid therapy, therapy should be discontinued with a gradual tapering off the opioid medication. ${ }^{1}$ Referral can be made to other specialists (eg, in the fields of psychiatry, orthopedics, and neurology) or to a drug abuse specialist/addictionologist, if needed. ${ }^{10}$

At the follow-up visit, the confirmatory results from the UDS are also examined to determine whether the patient has been compliant with the signed treatment agreement (presence of prescribed medication, absence of prohibited illicit substances). If UDS reports are positive for opioids other than those prescribed by the clinic, the source of the opioid is investigated with the patient. If the opioid was leftover from the previous physician, the patient is counseled and reminded not to repeat this infraction. If the opioid was prescribed by another physician, or if illegal substances are detected, the patient is terminated from treatment at the clinic and is mailed a letter stating the reasons for termination. Patients who are discharged are also offered names of other pain physicians who would consider taking them, as well as names of treatment facilities for abuse. In some instances, the clinic has continued to treat the patient's pain condition with nonscheduled medications or, on occasion, has enrolled a patient into the clinic's buprenorphine/naltrexone treatment program.

The clinic has a policy of not allowing early refills more than 5 days before schedule, or refills for lost or stolen medications. As a result, the clinic has not had any reported cases of lost or stolen medication or requests for early refills. The clinic has had similar success using this approach with patients taking morphine sulfate extended-release ${ }^{36}$ prior to the availability of MS-sNT.

\section{Costs and benefits of responsible monitoring for opioid-related aberrant behavior}

At the Pain Center of Devon, patients have not encountered difficulty with their health care plans and reimbursement for MS-sNT prescriptions. While the time required to manage any patient with chronic pain is substantial, perhaps the greatest material costs may be associated with the urine drug screening. As we believe that the qualitative on-the-spot UDS is indispensible in providing a snapshot of what to expect from each patient, the clinic pays for the point-of-service specimen cups, and the testing is typically reimbursed by Medicare and some private payers. In addition, the toxicology laboratory provides a person to perform collections at the center's office for quantitative analysis. This individual is responsible for collection and handling of the urine specimen, filling out associated forms, having the patient sign the specimen cup and form, and packing the specimens for shipment for quantitative analysis. The toxicology laboratory bills the insurance company directly. Additional time on the part of clinic personnel is required for receipt, interpretation and follow-up of results, and chart filing and handling; however, this time is well spent, as it enables us to provide responsible monitoring of patients so that problematic behaviors can be addressed immediately.

Outside of the clinic and community setting, misuse and abuse of opioids lead to substantial direct and indirect costs. Based on analysis of a claims database covering 2 million members from 16 large employers, between 1998 and 2002, opioid abusers aged between 12 and 64 years had higher usage rates of medical services, with total average per patient direct health payer costs estimated at US $\$ 15,884$, more than eightfold higher than that of nonopioid abusers at US\$1830 $(P<0.01) .{ }^{37}$ Additional costs result from consequent reduced productivity, prescription opioid theft, criminal activity, and strategies required to monitor, prevent, and deter misuse and abuse; however, there is limited information published about the costs of initiatives designed to reduce misuse, abuse, and diversion. A budget-impact model was used to estimate substantial savings to US third-party payers ranging from US $\$ 0.6$ billion to US\$1.6 billion per year resulting from introduction of a theoretical opioid formulation designed to resist or deter common methods of abuse. In addition, the public health consequences of opioid misuse and abuse would potentially be avoided. ${ }^{38}$

\section{Conclusion and future perspective}

Tailoring chronic pain management to individual patients, as is necessary with opioid therapy, ${ }^{1}$ may be best accomplished 
by combining evidenced-based medicine from clinical guidelines and practitioner experience. These cases demonstrate that several measures can enable the practitioner to both optimize pain-related outcomes and gain patient acceptance of formulations that incorporate a unique technology; these include the communication between physician and patient that accompanies complete pretreatment assessment, setting of treatment goals and expectations, proper instruction regarding the purpose and use of medications, and attention to individual patient issues. ${ }^{11}$ It is likely that in the future, most long-acting opioid preparations will include features designed to resist tampering, misuse, and abuse. Presumably, reduced likeability and tamper-resistance will decrease the desire and/or ability to abuse those opioid formulations by chewing, injecting, or snorting to obtain an instant high. Epidemiological studies will be needed to determine the impact of such formulations on misuse, abuse, and diversion of opioids in the community. In the meantime, communication and patient education are important to maximize the attainment of pain management goals while minimizing the risk of unintended consequences.

\section{Acknowledgments/Support}

Writing and editorial support was provided by Carol Berry and Jennifer Van Winckel at Quintiles Medical Communications, Parsippany, NJ, and funded by King Pharmaceuticals ${ }^{\circledR}$, Inc, which was acquired by Pfizer Inc in March 2011. Context and text are those of the author.

\section{Disclosure}

Daniel Rubino discloses that he has received no study grants or honoraria for this manuscript, and owns no stock in King Pharmaceuticals ${ }^{\circledR}$, Inc, or Pfizer Inc. He serves on the speaker bureau of King Pharmaceuticals ${ }^{\circledR}$, Inc, which was acquired by Pfizer Inc in March 2011, for EMBEDA ${ }^{\circledR}$ and FLECTOR ${ }^{\circledR}$ Patch. He has no other conflicts of interest to declare.

\section{References}

1. Chou R, Fanciullo GJ, Fine PG, et al. Opioid treatment guidelines. Clinical guidelines for the use of chronic opioid therapy in chronic noncancer pain. J Pain. 2009;10(2):113-130.

2. Gallagher RM, Rosenthal LJ. Chronic pain and opiates: balancing pain control and risks in long-term opioid treatment. Arch Phys Med Rehabil. 2008;89(3 Suppl 1):S77-S82.

3. McCarberg B, Stanos S. Key patient assessment tools and treatment strategies for pain management. Pain Pract. 2008;8(6):423-432.

4. Kuehn BM. Opioid prescriptions soar: increase in legitimate use as well as abuse. JAMA. 2007;297(3):249-251.

5. Substance Abuse and Mental Health Services Administration (SAMHSA). Results from the 2009 National Survey on Drug Use and Health: Volume 1. Summary of National Findings. Rockville, MD: SAMHSA; 2010.
6. Ives TJ, Chelminski PR, Hammett-Stabler CA, et al. Predictors of opioid misuse in patients with chronic pain: a prospective cohort study. $B M C$ Health Serv Res. 2006;6:46.

7. Fleming MF, Balousek SL, Klessig CL, Mundt MP, Brown DD. Substance use disorders in a primary care sample receiving daily opioid therapy. J Pain. 2007;8(7):573-582.

8. Sullivan MD, Edlund MJ, Fan MY, et al. Risks for possible and probable opioid misuse among recipients of chronic opioid therapy in commercial and medicaid insurance plans: the TROUP Study. Pain. 2010;150(2):332-339.

9. Passik SD, Messina J, Golsorkhi J, Xie F. Aberrant drug-related behavior observed during clinical studies involving patients taking chronic opioid therapy for persistent pain and fentanyl buccal tablet for breakthrough pain. J Pain Symptom Manage. 2010. [Epub ahead of print.]

10. Federation of State Medical Boards of the United States, Inc. Model Policy for the use of controlled substances for the treatment of pain. May 2004. Available from: http://www.fsmb.org/pdf.2004_grpol_ controlled_substances.pdf. Accessed December 21, 2010.

11. Gourlay DL, Heit HA, Almahrezi A. Universal precautions in pain medicine: a rational approach to the treatment of chronic pain. Pain Med. 2005;6(2):107-112.

12. Gourlay DL, Heit HA. Universal precautions revisited: managing the inherited pain patient. Pain Med. 2009;10 Suppl 2:S115-S123.

13. Brennan MJ, Stanos S. Strategies to optimize pain management with opioids while minimizing risk of abuse. PM R. 2010;2(6): 544-558.

14. Passik SD. Issues in long-term opioid therapy: unmet needs, risks, and solutions. Mayo Clin Proc. 2009;84(7):593-601.

15. Embeda [package insert]. Bristol, TN: King Pharmaceuticals ${ }^{\circledR}$, Inc. June 2009.

16. Stauffer J, Setnik B, Sokolowska M, et al. Subjective effects and safety of whole and tampered morphine sulfate and naltrexone hydrochloride (ALO-01) extended-release capsules versus morphine solution and placebo in experienced non-dependent opioid users: a randomized, double-blind, placebo-controlled, crossover study. Clin Drug Investig. 2009;29(12): 777-790.

17. Katz N, Sun S, Johnson F, Stauffer J. ALO-01 (morphine sulfate and naltrexone hydrochloride) extended release capsules in the treatment of chronic pain of osteoarthritis of the hip or knee: pharmacokinetics, efficacy, and safety. J Pain. 2010;11(4):303-311.

18. Johnson FK, Stark JG, Bieberdorf FA, Stauffer J. Relative oral bioavailability of morphine and naltrexone derived from crushed morphine sulfate and naltrexone hydrochloride extended-release capsules versus intact product and versus naltrexone solution: a single-dose, randomized-sequence, open-label, three-way crossover trial in healthy volunteers. Clin Ther. 2010;32(6):1149-1164.

19. Webster LR, Johnson FK, Stauffer J, Setnik B, Ciric S. Impact of intravenous naltrexone on intravenous morphine-induced high, drug liking, and euphoric effects in experienced non-dependent male opioid users. Drugs $R$ and D. 2011. [Epub ahead of print].

20. Jang DH, Robe JC, Hoffman RS. Severe opioid withdrawal due to misuse of new combined morphine and naltrexone product (Embeda). Ann Emerg Med. 2010;55(3):303-304.

21. Ruan X, Chen T, Gudin J, Couch JP, Chiravuri S. Acute opioid withdrawal precipitated by ingestion of crushed Embeda (morphine extended release with sequestered naltrexone): case report and the focused review of the literature. J Opioid Manag. 2010;6(4):300-303.

22. Oxycontin [package insert]. Stamford, CT: Purdue Pharma L.P.; April 2010.

23. OxyIR [package insert]. Stamford, CT: Purdue Pharma, L.P.; February 2007.

24. Passik SD, Kirsh KL, Whitcomb L, et al. A new tool to assess and document pain outcomes in chronic pain patients receiving opioid therapy. Clin Ther. 2004;26(4):552-561.

25. Percocet [package insert]. Chadds Ford, PA: Endo Pharmaceuticals, Inc; November 2006. 
26. Soma [package insert]. Somerset, NJ: Meda Pharmaceuticals, Inc; October 2009.

27. Colameco S, Coren JS. Opioid induced endocrinopathy. JAm Osteopath Assoc. 2009;109(1):20-25.

28. Rhodin A, Stridsberg M, Gordh T. Opioid endocrinopathy: a clinical problem in patients with chronic pain and long-term oral opioid treatment. Clin J Pain. 2010;26(5):374-380.

29. Katz NP, Adams EH, Chilcoat $\mathrm{H}$, et al. Challenges in the development of prescription opioid abuse-deterrent formulations. Clin J Pain. 2007;23(8):648-660.

30. American Academy of Pain Medicine, American Pain Society, American Society of Addiction Medicine. Definitions related to the use of opioids for the treatment of pain: a consensus statement for the American Academy of Pain, the American Pain Society, and the American Society of Addiction Medicine, 2001. Available from: http://www.ampainsoc.org/ advocacy/opioids2.htm. Accessed December 21, 2010.

31. American Academy of Pain Management. Opioid agreements/contracts: The American Academy of Pain Management's take on the subject

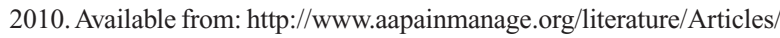
OpioidAgreements.pdf. Accessed December 21, 2010.
32. Birnbaum HG, White AG, Reynolds JL, et al. Estimated costs of prescription opioid analgesic abuse in the United States in 2001: a societal perspective. Clin J Pain. 2006;22(8):667-676.

33. Incidence and prevalence of ESRD. Am JKidney Dis. 1999;34(2 Suppl 1): S40-S50.

34. Knotkova H, Fine PG, Portenoy RK. Opioid rotation: the science and the limitations of the equianalgesic dose table. J Pain Symptom Manage. 2009;38(3):426-439.

35. Mercadante S, Bruera E. Opioid switching: a systematic and critical review. Cancer Treat Rev. 2006;32(4):304-315.

36. KADIAN (morphine sulfate extended-release) Capsules [package insert]. Morristown, NJ: Actavis Kadian LLC; February 2010.

37. White AG, Birnbaum HG, Mareva MN, et al. Direct costs of opioid abuse in an insured population in the United States. J Manag Care Pharm. 2005;11(6):469-479.

38. White AG, Birnbaum HG, Rothman DB, Katz N. Development of a budget-impact model to quantify potential cost savings from prescription opioids designed to deter abuse or ease of extraction. Appl Health Econ Health Policy. 2009;7(1):61-70. 


\section{Appendix}

\section{Sample Model Pain Management Agreement}

The purpose of this Agreement is to prevent misunderstandings about certain medicines you will be taking for pain mangement. This is to help both you and your doctor to comply with the law regarding controlled pharmaceuticals.

I understand that this Agreement is essential to the trust and confidence necessary in a doctor/patient relationship and that my doctor undertakes to treat me based on this Agreement.

I understand that if I break this Agreement, my doctor will stop prescribing these paincontrol medicines.

In this case, my doctor will taper off the medicine over a period of several days, as necessary, to avoid withdrawal symptoms. Also, a drug-dependence treatment program may be recommended.

I will communicate fully with my doctor about the character and intensity of my pain, the effect of the pain on my daily life, and how well the medicine is helping to relieve the pain.

I will not use any illegal controlled substances, including marijuana, cocaine, etc.

I will not share, sell or trade my medication with anyone.

I will not attempt to obtain any controlled medicines, including opioid pain medicines, controlled stimulants, or antianxiety medicines from any other doctor.

I will safeguard my pain medicine from loss or theft. Lost or stolen medicines will not be replaced.

I agree that refills of my prescriptions for pain medicine will be made only at the time of an office visit or during regular office hours. No refills will be available during evenings or on weekends.

I agree to use Pharmacy, located at

telephone number of my pain medicine.

I authorize the doctor and my pharmacy to cooperate fully with any city, state or federal law enforcement agency, including this state's Board of Pharmacy, in the investigation of any possible misuse, sale, or other diversion of my pain medicine. I authorize my doctor to provide a copy of this Agreement to my pharmacy. I agree to waive any applicable privilege or right of privacy or confidentiality with respect to these authorizations.

I agree that I will submit to a blood or urine test if requested by my doctor to determine my compliance with my program of pain control medicine.

American Academy of Pain Management • 13947 Mono Way \#A • Sonora, CA95370 • Phone: 209-533-9744 Fax: 209-533-9750 • e-mail: aapm@aapainmanage.org •www.aapainmanage.org

Appendix Figure I Sample model pain management agreement. ${ }^{3}$

Note: Reproduced with permission of the American Academy of Pain Management.

International Journal of General Medicine

Dovepress

\section{Publish your work in this journal}

The International Journal of General Medicine is an international, peer-reviewed open-access journal that focuses on general and internal medicine, pathogenesis, epidemiology, diagnosis, monitoring and treatment protocols. The journal is characterized by the rapid reporting of reviews, original research and clinical studies across all disease areas.
A key focus is the elucidation of disease processes and management protocols resulting in improved outcomes for the patient.The manuscript management system is completely online and includes a very quick and fair peer-review system. Visit http://www.dovepress.com/ testimonials.php to read real quotes from published authors. 